\title{
Polaronic effects in optical transitions of single InAs/AlAs quantum dots
}

\author{
D. Sarkar*, H.P. van der Meulen*, J.M. Calleja*, J.M. Becker ${ }^{\dagger}$, R.J. Haug ${ }^{\dagger}$ and \\ K. Pierz ${ }^{* *}$ \\ *Deptartamento Física de Materiales, Universidad Autónoma de Madrid, E-28049 Cantoblanco, Spain \\ ${ }^{\dagger}$ Institut für Festkörperphysik, Universität Hannover, D-30167 Hannover, Germany \\ **Physikalisch-Technische Bundesanstalt Braunschweig, D-38116 Braunschweig, Germany
}

\begin{abstract}
Optical transitions in self-assembled InAs/AlAs quantum dots were investigated by photoluminescence and photoluminescence excitation spectroscopy. In photoluminescence we found below about $1.8 \mathrm{eV}$ emission from quantum dots whereas above $\sim 1.8 \mathrm{eV}$ electrons from the AlAs X-band are involved. Photoluminescence excitation spectra show polaron states with a phonon energy of $31 \mathrm{meV}$ of an individual InAs dot.
\end{abstract}

\section{INTRODUCTION}

The InAs/AlAs material system is interesting for emission from quantum dots (QDs) at high energies. Optical properties of self-assembled QDs have been widely studied in InAs/GaAs systems, and an important feature is the polaronic coupling between discrete electronic states and phonons.

We present photoluminescence (PL) and PL excitation (PLE) results on InAs/AlAs single QDs revealing multiphonon sidebands. They are the consequence of the polaronic structure and give directly the value of the phonon frequency of the dot.

\section{EXPERIMENT}

The sample was grown on a GaAs(100) substrate by molecular beam epitaxy and consists of one layer of selforganized Stranski-Krastanov formed InAs QDs. The diameter of the dot is about $25-30 \mathrm{~nm}$, the height is about $2.5 \mathrm{~nm}$ and the density is about $3.6 * 10^{10} \mathrm{~cm}^{-2}$. The dots are embedded in an altogether $40 \mathrm{~nm}$ thick AlAs barrier. Finally the sample was capped by a $10 \mathrm{~nm}$ GaAs layer. By electron beam lithography openings of 0.2 to $10 \mu \mathrm{m}$ diameter were patterned in aluminium masks deposited on top of the sample, in order to select a few dots.

Measurements were performed in a liquid He continuous flow cryostat at $T=8 \mathrm{~K}$. For PL and PLE $\mathrm{Ar}^{+}$ and Ti:Sapphire lasers were used for sample excitation. Emission was collected through a 50x microscope objective, dispersed by a $0.85 \mathrm{~m}$ double grating spectrometer and detected with a nitrogen cooled CCD camera.

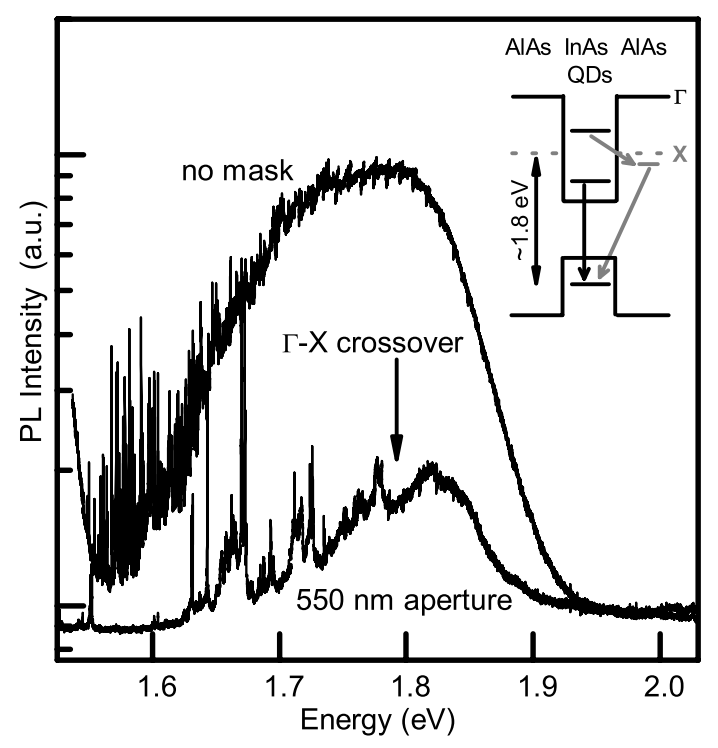

FIGURE 1. PL with and without mask. PL from QDs is observed only below $\sim 1.8 \mathrm{eV}$ as a consequence of the $\Gamma-X$ crossover with the AlAs barrier. Inset: Band sketch of the active region illustrating the two cases of recombination below and above $\sim 1.8 \mathrm{eV}$.

\section{RESULTS AND DISCUSSION}

Figure 1 compares PL spectra with and without mask, obtained using an excitation energy of $E_{\text {exc }}=2.41 \mathrm{eV}$. In both spectra sharp peaks corresponding to discrete dot levels are observed only below about $1.8 \mathrm{eV}$. Above that 


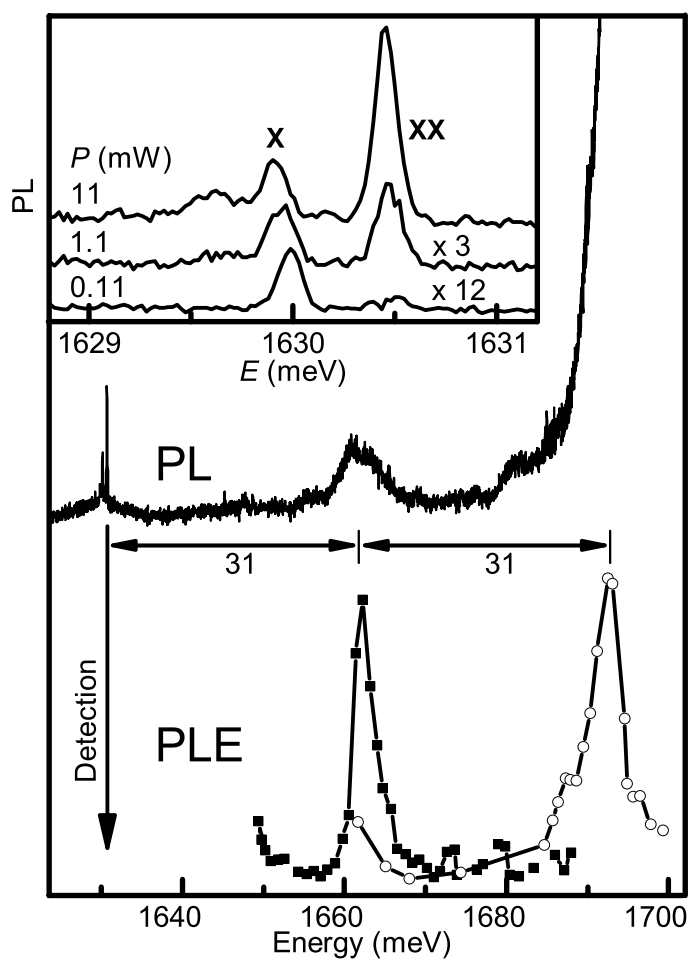

FIGURE 2. PL $\left(E_{\text {exc }}=1.71 \mathrm{eV}\right)$ and PLE spectra of the XX peak (see inset). PLE reveals polaron states, separated by the phonon frequency of the dot $(31 \mathrm{meV})$ which is close to the bulk InAs LO phonon frequency. Squares and cirles correspond to two different experimental runs. At $1 \mathrm{LO}$ the polaron state is also observed in PL $\left(E_{\text {exc }}=1.71 \mathrm{eV}\right)$. Inset: Power dependence of two peaks normalized to the $\mathrm{X}$ peak intensity. The peak XX is tentatively attributed to the biexciton.

energy they dissapear, as a consequence of the $\Gamma-X$ crossover with the AlAs conduction band. The electrons in small QDs with electronic ground states lying energetically above the X-band edge of the AlAs barrier can pass into the $\mathrm{X}$-band continuum and recombine with holes in the dot resulting in a continuous emission spectrum.

Micro PL with an excitation energy of $1.71 \mathrm{eV}$ of a selected single QD is shown in Fig. 2. The inset shows amplified the region around $1630 \mathrm{meV}$ with two peaks whose heights depend differently on pumping power. We tentatively attribute them to the exciton $(\mathrm{X})$ and biexciton (XX) transitions respectively.

PLE spectra of the XX line display two distinct peaks at 31 and $62 \mathrm{meV}$. The first peak can also be observed in PL as a broad emission when the sample is excited with an energy of $1.71 \mathrm{eV}$. They are attributed to polaron states of the dot [1]. Similar peaks have been observed in multi QD systems [2, 3] and in single InAs/GaAs QDs [4]. The $31 \mathrm{meV}$ spacing corresponds to the energy of the phonon forming the polaron. This is very close to the InAs LO phonon energy of $29.6 \mathrm{meV}$ considering hydrostatic deformation to match the AlAs lattice which shifts the phonon energy $+1.4 \mathrm{meV}$. The phonon of the dot can propagate into the AlAs barrier as twice the AlAs TA $(X)$ phonon energy of $13.5 \mathrm{eV}$ coincides approximately with the energy of the dot phonon. The width and structure of the 1LO and 2LO PLE peaks cannot be attributed to inhomogeneous broadening because we are detecting in a single dot. The detailed peak structure can be due to the hybridization of one electronic state with strong LO phonon satellites of another excited state [1]. The shoulder at $1688 \mathrm{meV}$ of the 2LO peak can also be originated by the overtone of the wetting layer phonon, whose energy is decreased by confinement.

\section{CONCLUSIONS}

In summary, we performed PL and PLE spectroscopy on InAs/AlAs QDs. The PL spectra can be divided in two regions: The part below about $1.8 \mathrm{eV}$ exhibits sharp peaks which arise from dot transitions. The continuous region above $\sim 1.8 \mathrm{eV}$ is due to recombination of $\mathrm{AlAs}$ $\mathrm{X}$-band electrons with dot holes.

In PLE measurements we have identified multi phonon sidebands of an electronic state of a single dot. The spacing of this polaron states reveals directly the phonon energy of the dot of $31 \mathrm{meV}$. This is close to the value of the InAs LO phonon energy taking into account hydrostatic strain.

This work was partially supported by the Spanish MCYT (MAT2002-00139), the CAM (07N/0042/2002) and the European network "Collect" (HPRN-CT-200200291).

\section{REFERENCES}

1. Seebeck, J., Nielsen, T. R., Gartner, P., and Jahnke, F., cond-mat/0406563 (2004).

2. Heitz, R., Veit, M., Ledentsov, N., Hoffmann, A., Bimberg, D., Ustinov, V., Kop'ev, P., and Alferov, Z. I., Phys. Rev. B, 56, 10435 (1997)

3. Hameau, S., Guldner, Y., Verzelen, O., Ferreira, R., Bastard, G., Zeman, J., Lemaître, A., and Gérard, J. M., Phys. Rev. Lett., 83, 4152 (2002).

4. Lemaitre, A., Ashmore, A. D., Finley, J. J., Mowbray, D. J., Skolnick, M. S., Hopkinson, M., and Krauss, T. F., Phys. Rev. B, 63, 161309 (2001). 
Copyright of AIP Conference Proceedings is the property of American Institute of Physics. The copyright in an individual article may be maintained by the author in certain cases. Content may not be copied or emailed to multiple sites or posted to a listserv without the copyright holder's express written permission. However, users may print, download, or email articles for individual use. 